\title{
The early immune response to infection of chickens with Infectious Bronchitis Virus (IBV) in susceptible and resistant birds
}

\author{
Jacqueline Smith ${ }^{1 *}{ }^{*}$, Jean-Remy Sadeyen ${ }^{2 \dagger}$, David Cavanagh ${ }^{2}$, Pete Kaiser ${ }^{1}$ and David W. Burt ${ }^{1}$
}

\begin{abstract}
Background: Infectious Bronchitis is a highly contagious respiratory disease which causes tracheal lesions and also affects the reproductive tract and is responsible for large economic losses to the poultry industry every year. This is due to both mortality (either directly provoked by IBV itself or due to subsequent bacterial infection) and lost egg production. The virus is difficult to control by vaccination, so new methods to curb the impact of the disease need to be sought. Here, we seek to identify genes conferring resistance to this coronavirus, which could help in selective breeding programs to rear chickens which do not succumb to the effects of this disease.

Methods: Whole genome gene expression microarrays were used to analyse the gene expression differences, which occur upon infection of birds with Infectious Bronchitis Virus (IBV). Tracheal tissue was examined from control and infected birds at 2, 3 and 4 days post-infection in birds known to be either susceptible or resistant to the virus. The host innate immune response was evaluated over these 3 days and differences between the susceptible and resistant lines examined.
\end{abstract}

Results: Genes and biological pathways involved in the early host response to IBV infection were determined andgene expression differences between susceptible and resistant birds were identified. Potential candidate genes for resistance to IBV are highlighted.

Conclusions: The early host response to IBV is analysed and potential candidate genes for disease resistance are identified. These putative resistance genes can be used as targets for future genetic and functional studies to prove a causative link with resistance to IBV.

Keywords: Chicken, Infectious bronchitis virus, Microarray, Disease resistance, Candidate gene

\section{Background}

Infectious bronchitis (IB) is a highly contagious respiratory disease of chickens first described in the USA in the 1930's [1-3]. Clinical signs include: coughing, sneezing, rales and nasal discharge. The disease can also affect the reproductive organs, which leads to a decrease in egg quality and production, thus making it a major cause of economic losses within the poultry industry [4]. The causative virus, Infectious Bronchitis Virus (IBV) is a coronavirus, which is an enveloped virus with a single positive-stranded RNA genome, which replicates in the

\footnotetext{
* Correspondence: Jacqueline.smith@roslin.ed.ac.uk

†Equal contributors

'The Roslin Institute \& R(D)SVS, University of Edinburgh, Easter Bush, Midlothian EH25 9RG, UK

Full list of author information is available at the end of the article
}

host cell cytoplasm [5]. Proteins encoded by IBV include the viral RNA polymerase, structural spike proteins, membrane and nucleocapsid and various other regulatory proteins. The spike glycoprotein mediates cell attachment and plays a significant role in host cell specificity [6].

The existence of many different IBV serotypes, which are not cross-protective means that control of IB, is very difficult. Mortality is usually fairly low $(\sim 5 \%)$, however some strains of the virus can also cause nephritis meaning that, depending on strain, mortality can be greater than $50 \%[7,8]$ or even up to $80 \%$ with some Australian isolates [9]. IBV infection leaves birds more susceptible to colibacillosis [10] and subsequent bacterial infections can also lead to a high level of mortality [11]. Currently, attenuated live vaccines are used in broilers and pullets, and 
killed vaccines are used in layers and breeders [12]. However, virus control is very difficult, as there are only a few vaccine types and many different strains of IBV. The virus also continues to mutate rapidly, generating more virulent strains of the disease [13-15]. Coronaviruses have now also been detected in other avian species such as turkey, duck, goose, pheasant, guinea fowl, teal, pigeon, peafowl and partridge [4].

The extent to which the virus affects the host is highly dependent on the chicken breed [4] and the MHC B locus is known to play a role in susceptibility to the virus [16]. In this study we attempt to identify non-MHC genes, which may be involved in resistance to IBV. No genetic analyses have thus far been undertaken in order to try and do this and no quantitative trait loci or genes associated with resistance have been determined, so far. Based on differential gene expression in susceptible and resistant lines of chickens, we identify potential candidate genes for disease resistance towards IBV (virulent M41 strain). Building on the previous work by Dar et al. [17] and Wang et al. [18] we used Affymetrix wholegenome chicken microarrays to examine the tracheal gene expression profiles of a line of birds known to be susceptible to IBV infection (line 15I) and a line known to show resistance (line $\mathrm{N}$ ). We determined the early host response to infection and propose possible candidate genes for involvement in disease resistance towards IBV. Understanding how coronaviruses infect the host and identifying genes involved in resistance is important not only for the poultry industry but also has important implications for human health, as diseases such as SARS are also caused by coronaviruses $[19,20]$.

\section{Methods}

\section{Ethics statement}

All animal work was conducted according to UK Home Office guidelines and approved by the Roslin Institute Animal Welfare and Ethical Review Body.

\section{Experimental animals}

The lines used in these experiments are an IBV susceptible line - line 15I (inbred White Leghorn strain) [21] and an IBV resistant line - line N (non-inbred Cornell strain). Line 15I was developed at East Lansing in the USA in the 1940s [22] and Line N at Cornell, USA in the 1960s [23]. The lines have since been maintained at the Institute for Animal Health in Compton, UK. Twoweek-old chicks from each line (15I and N) were separated into two experimental rooms, with ad libitum access to food and water. In one room, 54 birds (27 from each line) were infected with $4 \log _{10} \mathrm{CID}_{50}\left(10^{4} \mathrm{CID}_{50}\right)$ of virulent IBV-M41 strain in a total of $100 \mu \mathrm{l}$ of $0.2 \%$ BSA in PBS equally by intra nasal and ocular routes. In the other room, 54 control birds (27 from each line) received 100ul PBS via the same route. Trachea samples (upper half) were collected at 2, 3 and 4 days postinfection (9 individual birds from each line at each time point). The trachea of infected and control birds from each line were analysed for viral load using Taqman real-time quantitative RT-PCR assays.

\section{RNA preparation}

Tissue samples $(\sim 30 \mathrm{mg})$ were stabilized in RNAlater (Ambion, Life Technologies, Paisley, UK) and disrupted using a bead mill (Retsch MM 300, Retsch, Haan, Germany) at $20 \mathrm{~Hz}$ for $4 \mathrm{~min}$. Total RNA was prepared using an RNeasy kit (Qiagen, Crawley, UK) extraction method as per the manufacturer's protocol. Samples were resuspended in a final volume of $50 \mu \mathrm{l}$ of RNAsefree water. Concentrations of the samples were calculated by measuring $\mathrm{OD}_{260}$ and $\mathrm{OD}_{280}$ on a spectrophotometer (Nanodrop, Thermo Scientific, Paisley, UK). Quality of the RNA was checked on a bioanalyser (Agilent Technologies, South Queensferry, UK). An RNA integrity number $($ RIN) $>8$ proved the integrity of the RNA.

\section{Whole genome gene expression microarray hybridization} Biotinylated fragmented cRNA was hybridized to the Affymetrix Chicken Genome Array. This array contains comprehensive coverage of 32,773 transcripts corresponding to over 28,000 chicken genes. The Chicken Genome Array also contains 689 probe sets for detecting 684 transcripts from 17 avian viruses. For each experimental group (control and infected birds in each of the two lines at each of 2, 3 and $4 \mathrm{dpi}$ ), three biological replicates (3 RNA pools from 3 birds) were hybridized. Thus, 36 arrays were used in total. Hybridization was performed at $45^{\circ} \mathrm{C}$ for 16 hours in a hybridization oven with constant rotation $(60 \mathrm{rpm})$. The microarrays were then automatically washed and stained with streptavidin-phycoerythrin conjugate (SAPE; Invitrogen, Paisley, UK) in a Genechip Fluidics Station (Affymetrix, Santa Clara, CA). Fluorescence intensities were scanned with a GeneArray Scanner 3000 (Affymetrix, Santa Clara, CA). The scanned images were inspected and analyzed using established quality control measures. Array data have been submitted to Array Express (http://www.ebi.ac.uk/arrayexpress/) under the Accession Number E-TABM-1128.

\section{Statistical analysis}

Gene expression data generated from the GeneChip Operating Software (GCOS) was normalised using the PLIER (probe logarithmic intensity error) method [24] within the Affymetrix Expression Console software package. This normalised data was then analysed using the limma and FARMS [25] packages within $\mathrm{R}$ in Bioconductor [26]. Probes with a False Discovery Rate (FDR) 
Table 1 Primers used in QRT-PCR analysis

\begin{tabular}{|c|c|c|c|c|c|c|}
\hline Gene & Forward primer & Reverse primer & Probe primer & Opt. primer conc. & Probe $(\mu \mathrm{M})$ & GenBank \\
\hline $28 \mathrm{~S}$ & GGCGAAGCCAGAGGAAACT & GAC GACCGATTTGCACGTC & AGGACCGCTACGGACCTCCACCA & 0.6 & 5 & FM165415 \\
\hline C1S & GCGCAAAGGCTGGAAAATAC & TCAAGAACAGAATTGGGAGTGACA & TACTATGCTGAACCCATAACCTGTCTCCCG & 0.6 & 5 & NM_001030777 \\
\hline CCL13 & CAGAGCCTGGCCCAGAGA & TGTCCATTTGATTCTTCTGGTATG & CTGTGCCTGACAAGTGCTGCTTCAACTT & 0.2 & 5 & XM_415779 \\
\hline CCLi7 (ah221) & САCAACCTGCTGCTTCTCCTATG & TGTAGGCGGAGGCAATGAG & TCAACGTCCCGTCCCACGCA & 0.2 & 5 & AY037860 \\
\hline CD38 & GCTTGATGGGCTTTCATGGT & CACATTCACTCCATTTTGGACAA & ACCCCTCAGCTCCAGGAATCAACTATGAA & 0.6 & 5 & XM_420774 \\
\hline CLU & TGAGTCAGAATCCCGTAACTTCAG & GCAGTCCACAGCCAAGATCTC & AGATCCGGCGCAACTCGGCC & 0.2 & 5 & NM_204900 \\
\hline $\operatorname{cox} 11$ & TGGGATCTCCACCTACAACGTA & CAGCCACTGTTCTTCAAAACAAA & TGCCCTTCGAAGCAGGACAGTACTTCA & 0.4 & 5 & XM_001233972 \\
\hline DDT & GGCCCCGAGCGGATT & CATGACTGTTCTGTTCTTGCCAAT & CATTCGCTITTACCCGCTGGAGCC & 0.8 & 5 & NM_001030667 \\
\hline FK-506-BP51 & CGGAGGATCAAGAGGAAAGGA & CAGAACCCCTCCAGGTGAATT & AAGGCTATTCCAACCCCAACGAAGGTG & 0.1 & 5 & NM_001005431 \\
\hline HSC2O & GGAAATCATGGAAATCAATGAGAAA & CACCTCTTTGGTCAGTTCTTCTTG & CAGAGCCCGAGAACGACGAGATCC & 0.8 & 5 & XR_026662 \\
\hline IFNAR2 & TGGTCACTGCATCTCTAAATAAACATT & CTGCAATTGTGATGCCATAATAATC & CATCCCATCAGCCTGGAAATGCATAACT & 0.4 & 5 & NM_204858 \\
\hline IGFBP5 & GAAGAGCAGCCAGAGGATGGT & GCTTGCACTGCTTCCTCTTGT & CACCTCCCCAACTGCGACCGAAA & 0.1 & 5 & XM_422069 \\
\hline IRF-7 & ACCCGGACCGCCGTAT & GCCCAGGCCTTGAAGATCTC & CATCCCTTGGAAGCACAACGCCA & 0.6 & 5 & NM_205372 \\
\hline MAFK & GCGATGATGAACTCGTGTCAA & TTCAGACGGATGACCTCCTCTT & TCCGTACGGGAGCTGAACCAGCAC & 0.4 & 5 & NM_204756 \\
\hline MAP4K4 & TGCTATTGAAATGGCTGAAGGA & TCCGTGGGATGAGGAAGAGT & TCCTCCCCTGTGTGACATGCACCC & 0.2 & 5 & NM_001031126 \\
\hline MMD2 & TGCCACGCACGCATTCT & GGTCATCGGAGAGGACGTAGAG & TCCTGCCCAGCATCCTCGGC & 0.4 & 5 & XM_414787 \\
\hline MX1 & TGGACTTCTGCAACGAATTGTC & ATCCAGAAGAGTGCTGAAATGTTTG & TTCACCTCCGCAATCCAGCAAGAGA & 0.6 & 5 & NM_204609 \\
\hline SRI & TACTATCAGGGCGGGTATGGA & AGCAAAATAACCATACAGAGGATCCT & CAGCTCCAGGAGGCCCATCATTCC & 0.1 & 5 & NM_001080865 \\
\hline SUCLG2 & AGCTTCCCGGCTGTTCAAT & CATGGTCTGCCATCAGCTTTT & AACCCCTAGACGATGGCTGAATCTGCA & 0.1 & 5 & NM_001006141 \\
\hline TLR3 & ATCCATGGTGCAGGAAGTTTAAG & GATGGAGTCTCGACTTTGCTCAATA & TGCATCATGCTTTACAGC & 1.0 & 5 & $J F 273967.1$ \\
\hline TNFAIP1 & GTTGTGGGAAGCACTTTGGAA & TCAACTCCTTGATCTCCTGTCTGT & CCGAGATGACACAATTGCACTTCCAAAA & 0.2 & 5 & NM_001030726 \\
\hline TP-D53 & TGGCCAAGCTAGAGGATGAAA & TCAGGCTCATGCCAAGTTTT & ACTAGCAGCCAAAGAAAAGCACCTGATTGA & 0.2 & 5 & NM_204215 \\
\hline
\end{tabular}


value $<0.05$ and a fold change $\geq 1.5$ were deemed to be biologically significant.

\section{Analysis of differentially-expressed genes}

In order to determine which biological pathways are involved in the responses to viral infection, we analysed our differentially-expressed (DE) genes using Pathway Express [27, 28] which uses KEGG pathways [29] to pictorially display up/down regulation of genes. (NB. These diagrams are based on the human pathways and so are not completely representative of the chicken pathways). Genes differentially expressed during the host response $($ FDR $<0.05)$ were analysed against a reference background consisting of all genes expressed in the experiment. Factors considered by Pathway Express include the magnitude of a gene's expression change and its position and interactions in any given pathway, thus including an 'impact factor' when calculating statistically significant pathways. Anything with a $p$-value $<0.25$ is deemed significant when using this software. Use of the Ingenuity Pathway Analysis (IPA) program [30] revealed which canonical pathways are being switched on by IBV infection in the host (with Benjamini-Hochberg multiple testing correction) and allowed us to analyze the gene interaction networks involved in the host response. Genes were clustered by similar expression pattern and analysed for enriched GO-terms and transcription factor binding sites (TFBS) using Expander (v5.2) [31]. Normalised expression data from control samples were compared with infected samples to examine the host response to IBV infection. Enrichment analysis of particular GO terms or TFBS within clusters was done using the TANGO and PRIMA functions, respectively, within the Expander package.

\section{Viral quantification and specific gene expression analysis} by quantitative real-time PCR

Taqman real-time quantitative RT-PCR (qRT-PCR) was used to quantify viral RNA levels and for confirmation of the microarray results for the mRNA levels of selected genes. This was performed on 3 replicate pools of 3 samples (9 birds). Primers (Sigma) and probe (PE Applied Biosystems, Warrington, UK) (Table 1) were designed using Primer Express (PE Applied Biosystems). Briefly, the assays were performed using $2 \mu \mathrm{L}$ of total RNA and the Taqman FAST Universal PCR Master Mix and one-step RT-PCR mastermix reagents (PE Applied Biosystems) in a $10 \mu \mathrm{L}$ reaction. Amplification and detection of specific products were performed using the Applied Biosystems 7500 Fast Real-Time PCR System with the following cycle profile: one cycle at $48{ }^{\circ} \mathrm{C}$ for $30 \mathrm{~min}$ and $95{ }^{\circ} \mathrm{C}$ for $20 \mathrm{sec}$, followed by 40 cycles at $95{ }^{\circ} \mathrm{C}$ for $3 \mathrm{sec}$ and $60{ }^{\circ} \mathrm{C}$ for $30 \mathrm{sec}$. Data are expressed in terms of the cycle threshold $(\mathrm{Ct})$ value, normalised for each sample using the $\mathrm{Ct}$ value of $28 \mathrm{~S}$ rRNA product for the same sample, as well described previously [32-34]. Final results are shown as 40-Ct using the normalised value, or as fold-change from uninfected controls.

\section{Results and discussion}

\section{Assessment of viral load}

Taqman real-time quantitative RT-PCR analysis was used to measure viral load in trachea samples from both control and infected birds from both lines $15 \mathrm{I}$ and $\mathrm{N}$. Tracheal tissue was chosen for examination in this study as the target of IBV is the epithelial surface of the respiratory tract. Viral RNA was detected in infected birds, but no significant difference in viral load was detected between lines at any of the days 2, 3 or 4 post infection (Fig. 1). This would indicate that the resistance to the virus seen in Line $\mathrm{N}$ is due to how the birds respond to the virus once it has entered the body and is not a measure of how the birds can prevent initial infection by the virus itself. When resistance to IBV infection was originally determined in these lines, it was noted that they were equally susceptible to infection, but a variation in outcome was seen. In line N, $33 \%$ of birds showed air sac lesions whereas $73 \%$ of $15 \mathrm{I}$ birds presented lesions. Mortality was 0 in line N, but $47 \%$ within line $15 \mathrm{I}$ birds. It was hypothesized that the different lines were producing different immunological responses upon infection [21].

\section{Host response to IBV infection}

Gene expression differences found in the susceptible 15I line between infected and control birds over days 2, 3 and 4 post infection were analysed, with a view to examining the innate host response to infection by IBV. Genes seen to be induced during the host response to infection include C1S, IRF1, STAT1, MX1, TLR3 and CTSS as previously recognised by Guo et al. [35]. We also identified IFIT5, OASL, SCA2, LYG2, ISG12-2, DDX60, IFIH1, IRF7,

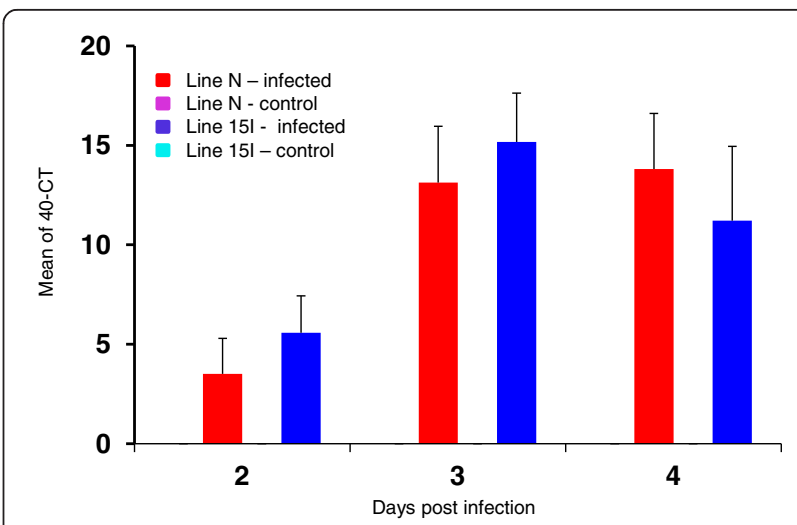

Fig. 1 QRT-PCR measurement of viral load in control and infected birds from lines 151 and N. Mean 40-Ct values are shown with the standard error mean indicated 
ZC3HAV1, DHX58, CCli7, IFITM1 and IFITM3 as being up-regulated in response to IBV infection. Few genes are seen to be down-regulated during the early stage of the host response, but those which are include $C H A C 1$ (pro-apoptotic), $H B B$ (implicated in inflammation regulation) and $P D K 4$ (glucose regulation). For a full list

a ANTIGEN PROCESSING AND PRESENTATION

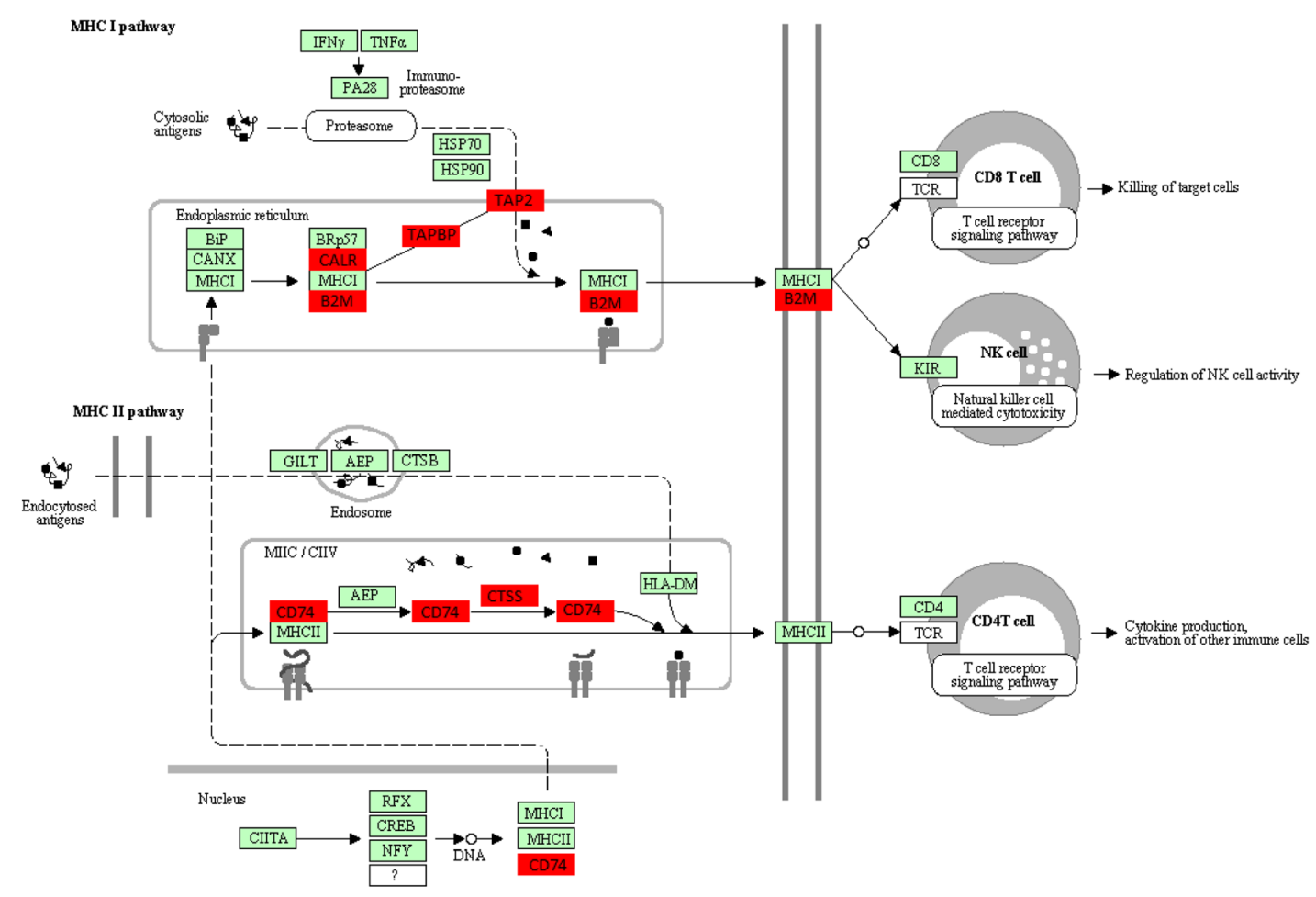

b TOLL-LIKE RECEPTOR SIGNALING PATHWAY

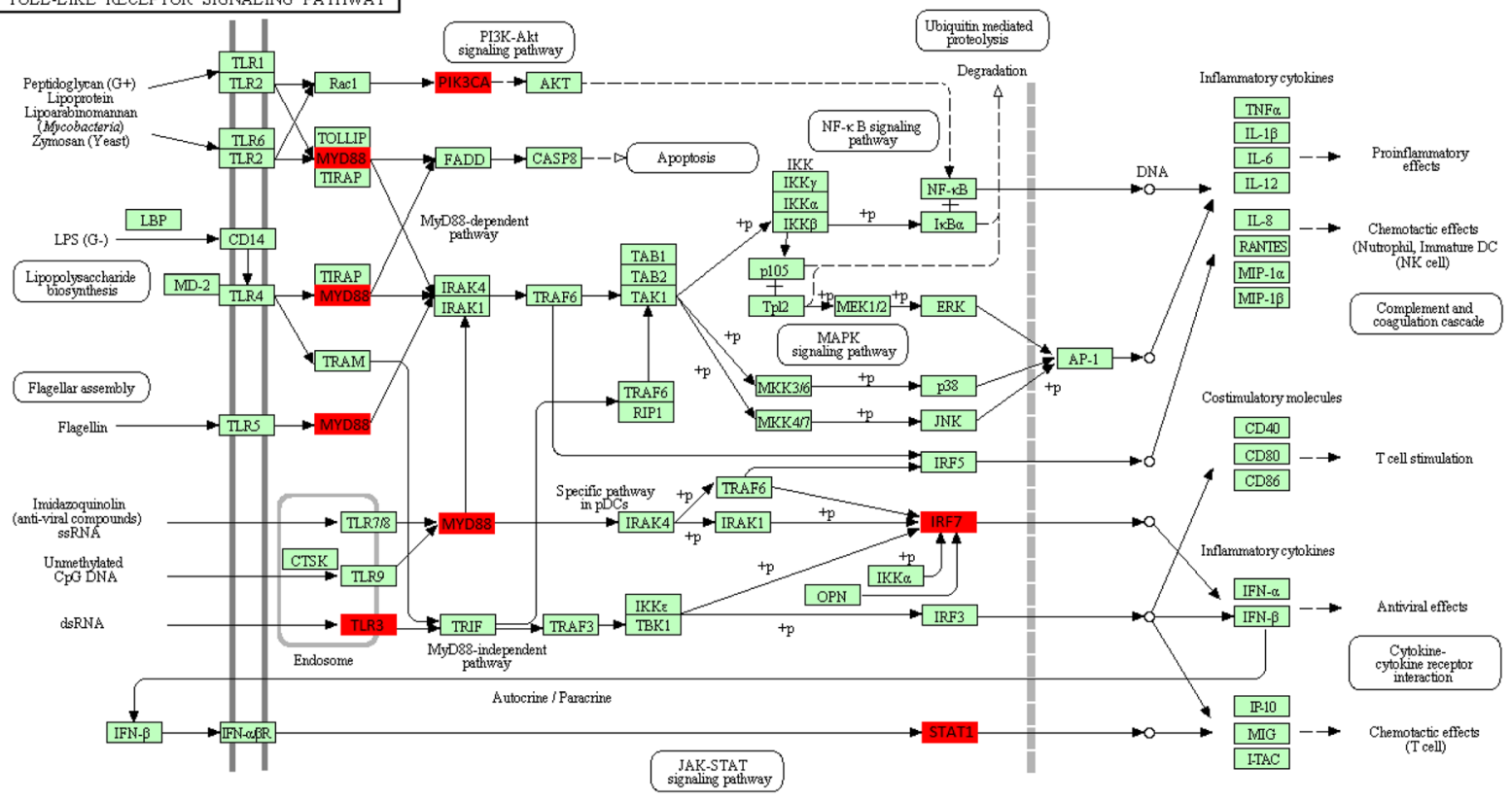

Fig. 2 Pathway Express analysis of the host response to IBV infection in the trachea of susceptible birds (Line 15I). Many genes involved in antigen processing and presentation (a) and in the Toll-like receptor pathway (b) can be seen to be up-regulated (shown in red) 
Table 2 Pathway Express analysis of the host response to IBV infection

\begin{tabular}{lllll}
\hline Rank & Pathway name & Impact Factor & Input genes/Genes in Pathway & Corrected gamma $p$-value \\
\hline 1 & Antigen processing and presentation & 14.244 & $6 / 89$ & $9.93 \mathrm{E}-06$ \\
2 & Toll-like receptor signaling pathway & 7.927 & $5 / 102$ & 0.003221463 \\
3 & Notch signaling pathway & 7.073 & $3 / 47$ & 0.006843371 \\
4 & Pancreatic cancer & 4.448 & $3 / 72$ & 0.06375221 \\
5 & Maturity onset diabetes of the young & 3.952 & $1 / 24$ & 0.095158775 \\
6 & Phosphatidylinositol signaling system & 3.884 & $2 / 76$ & 0.100456001 \\
7 & Complement and coagulation cascades & 3.841 & $3 / 69$ & 0.103946526 \\
8 & mTOR signaling pathway & 3.652 & $2 / 52$ & 0.120669151 \\
9 & Acute myeloid leukemia & 3.448 & $2 / 59$ & 0.141487282 \\
10 & Systemic lupus erythematosus & 3.332 & $2 / 144$ & 0.15474593 \\
11 & VEGF signaling pathway & 3.126 & $2 / 74$ & 0.181102595 \\
\hline
\end{tabular}

of the genes involved in the tracheal immune response (133 DE probes), see Additional file 1: Table S1.

To elucidate which biological pathways are being perturbed during the host response to IBV infection, we analysed our data using Pathway Express [36]. The resulting pathway diagrams are extremely useful in establishing which gene networks are involved in a particular experimental response. As seen in Fig. 2, genes involved in antigen presentation and the Toll-like receptor (TLR) pathway are up-regulated. TLRs identify pathogen associated molecular patterns (PAMPs) and are crucial to the innate immune system. In this study TLR3 is shown to be
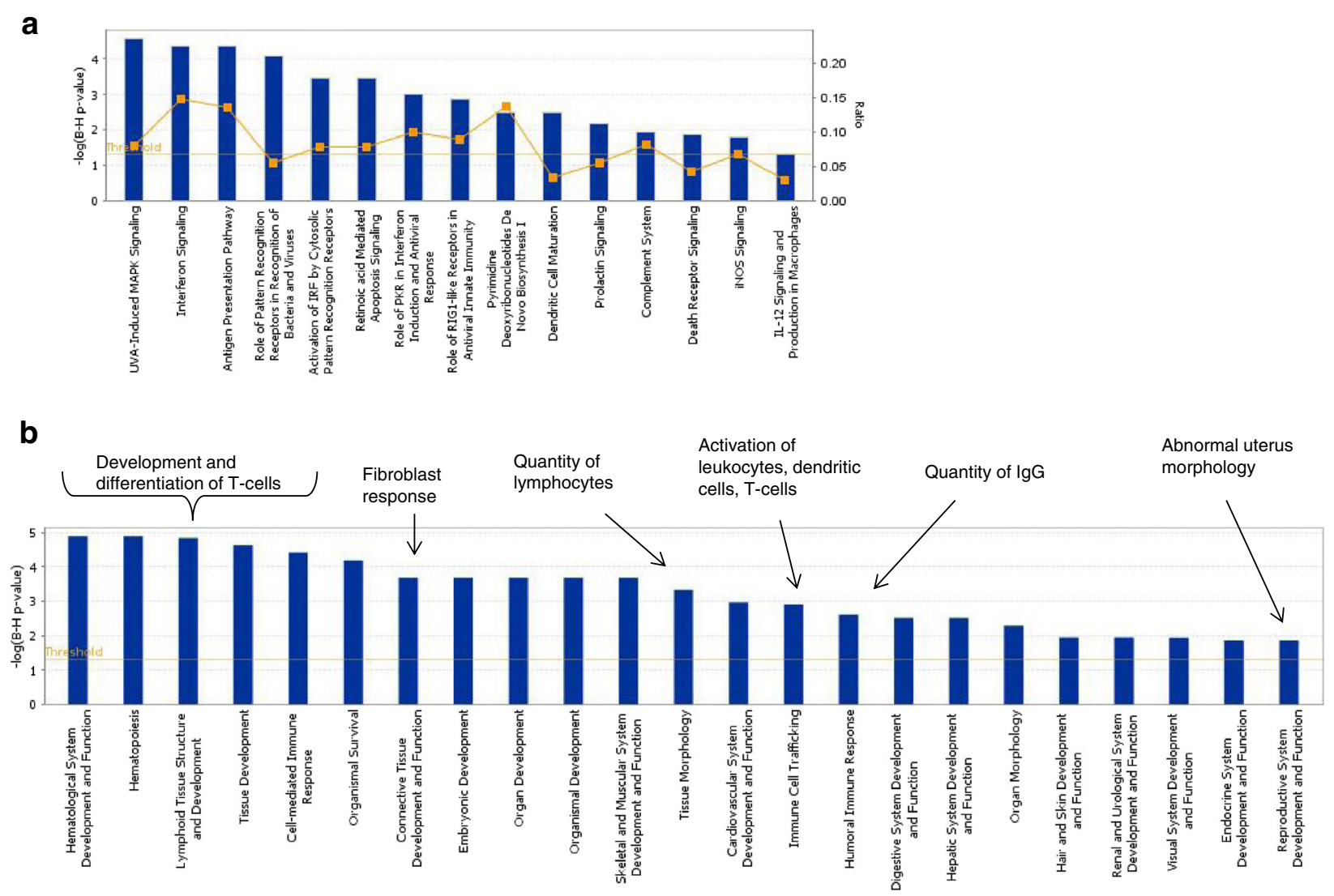

Fig. 3 Ingenuity Pathway Analysis (IPA) of genes responding to IBV infection. a Canonical biological pathways which are activated in the host upon IBV infection $(p<0.05)$. The line represents the ratio of genes represented within each pathway. $\mathbf{b}$ The most highly represented $(p<0.05)$ physiological functions of genes differentially expressed during the host response to IBV (in the trachea in susceptible birds (Line 15I)). Specific functions within groups are highlighted 


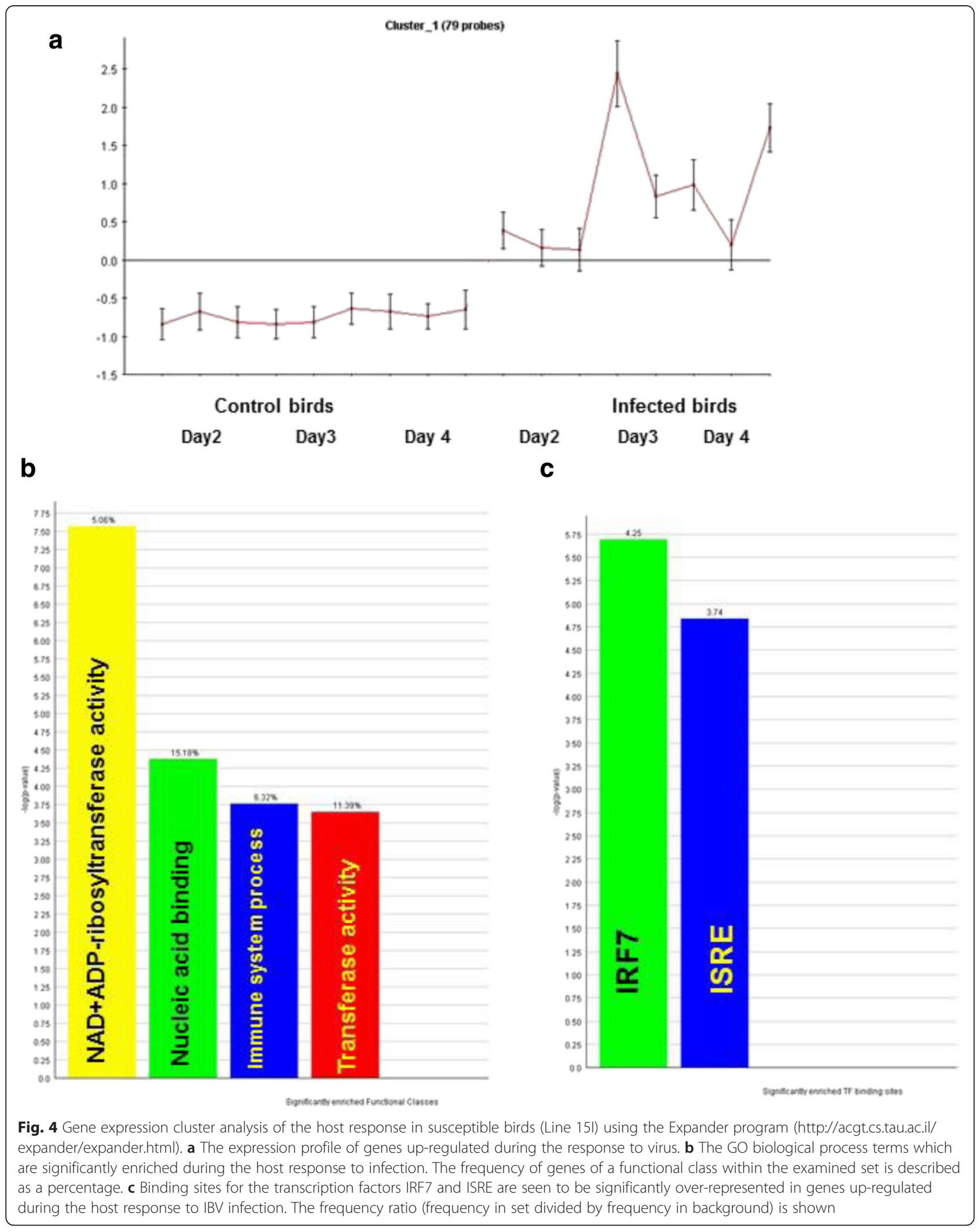


induced at 3 dpi. TLR3 recognizes double-stranded RNA intermediates produced during viral replication and has previously been shown to be induced in the trachea at this time after IBV infection [37]. Another pathway involved is the phosphatidylinositol signalling pathway (Table 2). Phosphatidylinositol kinases are known to play an important role in the viral life cycle after infection of the host and PI4KB is known to be exploited by coronaviruses for viral entry. The product of PI4KB catalysis is phosphatidylinositol 4-phosphate (PI4P) and coronavirus entry into the host is mediated by the PI4P lipid microenvironment [38]. Genes involved in the complement system are also highlighted as being up-regulated in response to IBV infection. Complement-mediated lysis of viruses is an important facet of the host innate immune system and its role in defence against viral infection [39] - as reflected in the induction of these genes in this study.

Use of Ingenuity Pathway Analysis (IPA) software also allowed us to determine which biological systems are active during the host response. Up-regulated genes are seen to be part of the canonical biological pathways shown in Fig. 3a. Biological processes involving pattern recognition receptors and interferon signalling feature heavily. The interferon response is a powerful antiviral mechanism, which has previously been shown to be involved in the host response after IBV infection. A very early induction of IFN- $\gamma$ has been reported in splenocytes [40], and in peripheral blood mononuclear cells (PBMCs) and lung leukocytes [41]. IFNB expression has also been reported in trachea between 1 and 2 dpi [42]. We do not see this increase in expression of interferon genes (due to the absence of data earlier than $2 \mathrm{dpi}$ ), but we do see the downstream effects, with increased expression of many interferon-induced genes. Specific physiological processes activated upon IBV infection can also be seen in Fig. 3b. The stimulation of various different immune cells is seen along with the indication of reproductive abnormality, which would reflect the problems seen with egg-laying upon IBV infection.
In order to cluster genes seen to be involved in the host response to infectious bronchitis into groups with similar expression profiles and probably sharing similar functions or gene regulatory pathways, we utilised the CLICK algorithm within the Expander program [43]. Figure 4a shows the expression profile of genes upregulated during the response to virus. The Expander program was also used to analyse the Gene Ontology (GO) functional annotations of the genes being differentially expressed. Figure $4 \mathrm{~b}$ shows the biological process terms, which are significantly enriched in the genes responding during the host response to infection. As would be expected, these include terms like 'innate immune response' and 'antigen processing and presentation'. 'NAD + ADP-ribosyltransferase activity' and 'phosphoinositide binding' are also highlighted. Transcription factor binding sites present in DE genes which are significantly over-represented were also predicted. Figure $4 \mathrm{c}$ shows that genes up-regulated during the host response have a high proportion of IRF7 and ISRE binding sites. IRF7 is a transcriptional activator, which binds to the interferon-stimulated response element (ISRE) in IFN promoters and functions as a molecular switch for antiviral activity.

\section{Differences between susceptible and resistant lines}

Analysis of the gene expression differences between infected and control birds across the two lines has provided us with information on how these lines differ in their response to infection. Examination of the gene expression profiles in the control birds of the two different lines also allowed us to identify genes, which are inherently different between the susceptible and resistant birds. It can be seen that there are numerous genes, which show large expression differences between the two lines, even before infection. Dramatic differences in gene expression of certain genes, including $D D T, S R I$, $B L B 1, H S C B, B F 1, B F 2$, SUCLG2, MX1 and SRI, which are more highly expressed in the resistant $\mathrm{N}$ line

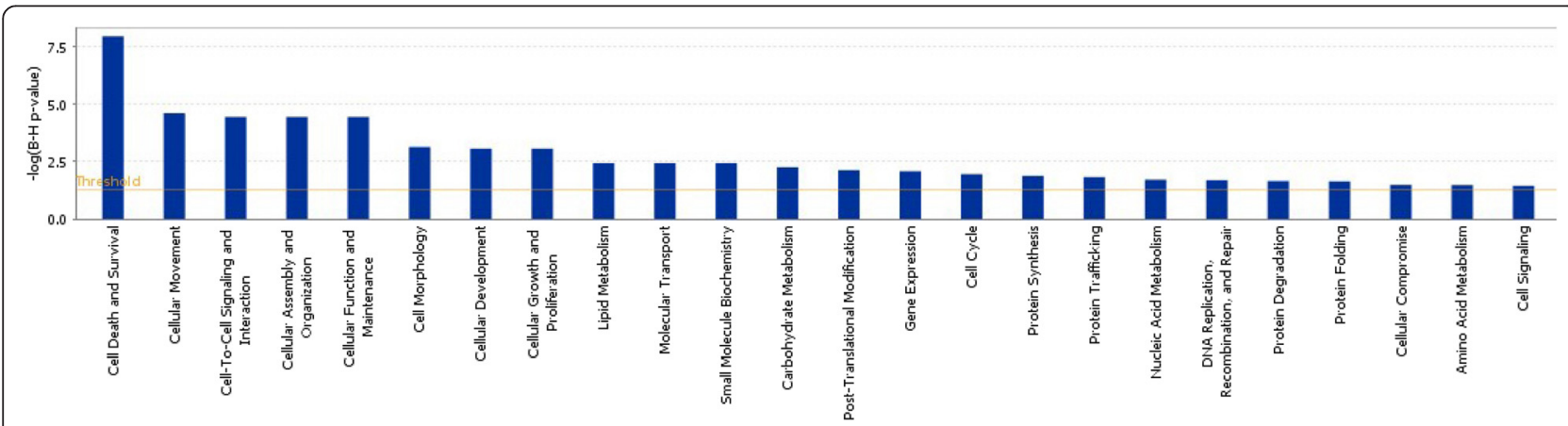

Fig. 5 Ingenuity Pathway Analysis (IPA) of genes showing inherent differential expression between susceptible and resistant control birds. This graph shows the most highly represented $(p<0.05)$ molecular functions of DE genes 


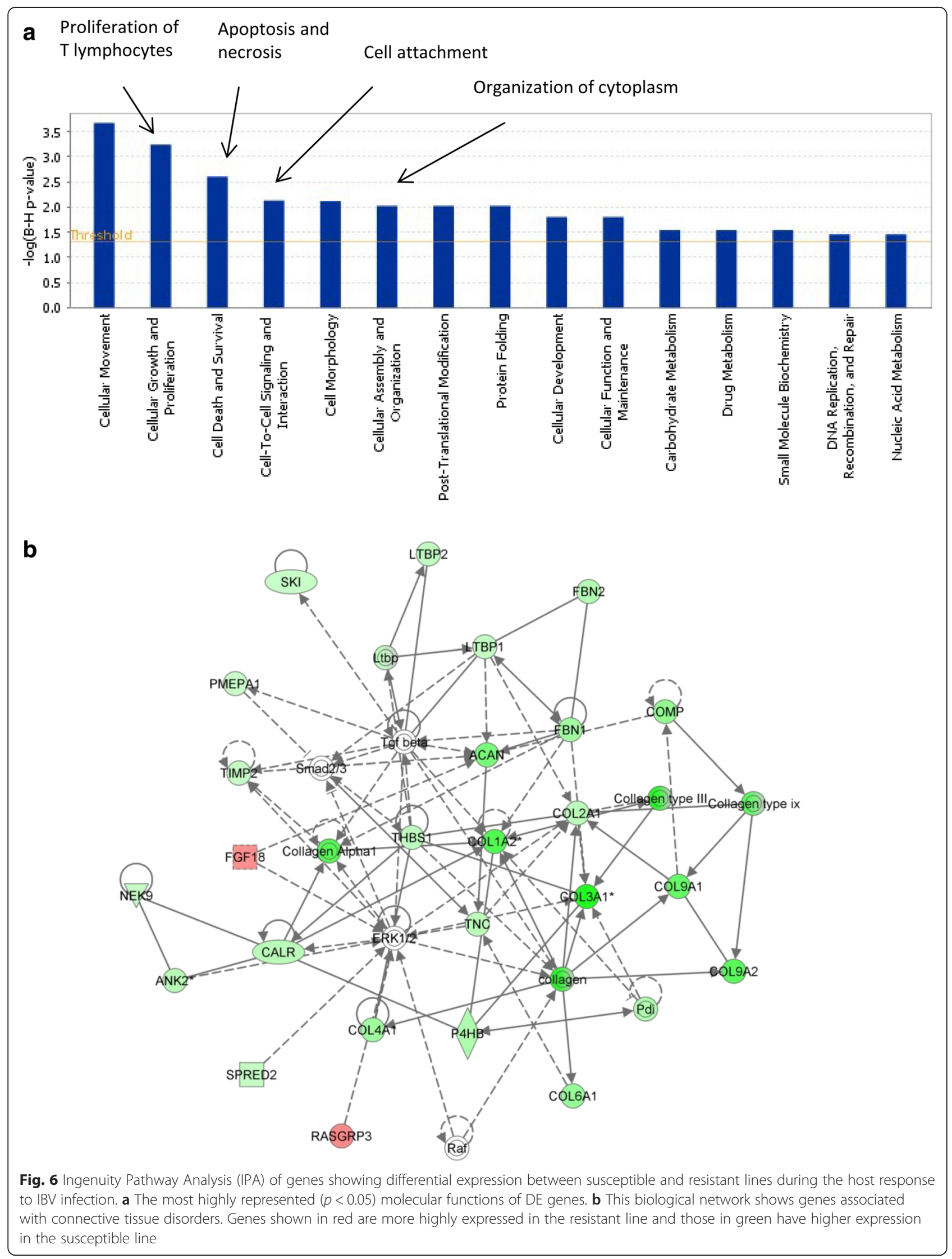


compared to the susceptible 15I line are noted (Additional file 2: Table S2 shows all $1930 \mathrm{DE}$ probes) So, it can be seen that these are genes which have inherently different expression levels between susceptible and resistant birds, even before infection occurs. We therefore postulate that some of these genes may play an important role in disease resistance. The potential interactome of IBV has recently been investigated by stable isotope labelling with amino acids in cell culture (SILAC) coupled to a green fluorescent proteinnanotrap pull-down methodology [44]. Host proteins, which bind to the IBV $\mathrm{N}$ protein were identified, some of the genes for which, we see as being inherently expressed at higher levels in susceptible birds in this study. These genes include MYH9, CAPRIN1, DHX57, HNRNPH3, RPL27A, FMR1, C22orf28, HNRPDL, SFRS3, RPL31, NPM1 and RPSA. This may therefore be one of the reasons why Line $15 \mathrm{I}$ is more susceptible to IBV infection - there are more host proteins to which the virus binds, compared with the resistant Line $\mathrm{N}$.

Upon infection, differences in response are also seen between the two lines. Interestingly, apart from CD38 and $C D 4$ at 3 dpi and FKBP5 at $4 \mathrm{dpi}$, all other differential gene expression between the lines is seen at $2 \mathrm{dpi}$ in this study (Additional file 3: Table S3). CD38 is a glycoprotein found on the surface of many immune cells including
CD4+, CD8+, B lymphocytes and natural killer cells and is a marker of cell activation. It functions in cell adhesion, signal transduction and calcium signalling. CD4 is found on the surface of immune cells such as $\mathrm{T}$ helper cells, monocytes, macrophages and dendritic cells. It is a membrane glycoprotein which interacts with MHCII antigens. The protein functions to initiate or augment the early phase of T-cell activation. The protein encoded by FKBP5 is a member of the immunophilin protein family, which play a role in immuno-regulation and basic cellular processes involving protein folding and trafficking. Early defence by the host is a key mechanism for combatting viral infection, and induction of IFNB and other innate genes in response to IBV infection has been shown to peak around 18-36 hr post infection [42].

In this study, genes more highly expressed (or less down-regulated) in the resistant $\mathrm{N}$ line at $2 \mathrm{dpi}$ include a number of collagen genes (COL3A1, COL1A2, COL9A1, COL9A2, COL6A1 and COL4A1) and other genes such as ACAN, FSTL1, COMP, EIF3A, STAT3 and IGFBP5. Genes seen to be more highly expressed (or less down-regulated) in the susceptible 15I line include RBM39, MAFB, NNK2, CCN1, MGAT5 and THRAP3. One consequence of IBV infection is the production of poor quality, misshapen eggs by infected birds [45]. Some of the genes previously identified as being important for the creation of a

Table 3 Genes analyzed by qRT-PCR

\begin{tabular}{|c|c|c|c|c|}
\hline Gene & Description & Fold change & GenBank & Ensembl \\
\hline MX1 & Interferon-induced GTP-binding protein Mx & $6-20$ & NM_204609 & ENSGALG00000016142 \\
\hline C1S & complement component 1, s subcomponent & $2-4$ & NM_001030777 & ENSGALG00000014603 \\
\hline IRF7 & Interferon regulatory factor 7 (IRF-7) & $4-5$ & NM_205372 & ENSGALG00000014297 \\
\hline TLR3 & Toll-like receptor 3 & 4 & NM_001011691 & ENSGALG00000013468 \\
\hline CCLi7 & Chemokine ah221 & $2-12$ & AY037860 & ENSGALG00000002343 \\
\hline CD38 & ADP-ribosyl cyclase 1 & $4-9$ & XM_420774 & ENSGALG00000014508 \\
\hline FKBP5 & FK-506 binding protein 51 & $5-6$ & NM_001005431 & ENSGALG00000000947 \\
\hline IGFBP5 & Insulin-like growth factor binding protein 5 & 1 & XM_422069 & ENSGALG00000011468 \\
\hline DDT & D-dopachrome tautomerase & $2-3$ & NM_001030667 & ENSGALG00000006350 \\
\hline SRI & sorcin & 2 & NM_001080865 & ENSGALG00000008985 \\
\hline CLU & clusterin & 2 & NM_204900 & ENSGALG00000016587 \\
\hline $\operatorname{cox} 11$ & Cytochrome c oxidase assembly protein COX11, mitochondrial precursor & 2 & XM_001233972 & ENSGALG00000003017 \\
\hline MMD2 & Monocyte to macrophage differentiation factor 2 & 2 & XM_414787 & ENSGALG00000004530 \\
\hline IFNAR2 & interferon alpha/beta receptor 2 & $2-3$ & NM_204858 & ENSGALG00000015938 \\
\hline TNFAIP1 & tumor necrosis factor, alpha-induced protein 1 (endothelial) & 2 & NM_001030726 & ENSGALG00000005715 \\
\hline MAP4K4 & mitogen-activated protein kinase kinase kinase kinase 4 & 2 & NM_001031126 & ENSGALG00000008970 \\
\hline MAFK & Transcription factor MafK. & 2 & NM_204756 & ENSGALG00000004189 \\
\hline CCL13 & similar to Small inducible cytokine A13 precursor (CCL13) & 3 & XM_415779 & ENSGALG00000024470 \\
\hline TPD52L1 & Tumor protein D53 homolog & $13-23$ & NM_204215 & ENSGALG00000014843 \\
\hline $\mathrm{HSCB}$ & Co-chaperone protein $\mathrm{HscB}$, mitochondrial precursor $(\mathrm{Hsc} 20)$ & 2 & XR_026662 & ENSGALG00000005706 \\
\hline SUCLG2 & succinate-CoA ligase, GDP-forming, beta subunit & 2 & NM_001006141 & ENSGALG00000007652 \\
\hline
\end{tabular}


healthy eggshell are seen to be more highly expressed by the resistant $\mathrm{N}$ line birds after infection in this study. These genes include COL1A2, CRELD2, HSP9OB1, $P 4 H B$ and ERP29 [46]. For a full list of genes differentially expressed between the two lines in trachea (409 DE probes) see Additional file 3: Table S3.

IPA analysis of genes showing different inherent expression between lines $15 \mathrm{I}$ and $\mathrm{N}$ shows that the molecular functions of these genes is primarily concerned with their involvement in cell death and cell adhesion (Fig. 5), two processes previously shown to be significant in infected kidneys [47]. When the differential host responses to infection are examined, it is seen that genes involved in proliferation of T-lymphocytes and genes concerned with cell attachment and cytoplasmic organization are more highly expressed in the resistant line N. Other processes significantly involved are apoptosis and necrosis (Fig. 6a), which have been previously documented in IBV-infected Vero cells by Liu et al. [48].
One of the most perturbed biological networks noted in this analysis is that involving genes related to connective tissue disorders and involve many collagen genes. These genes are more highly expressed in susceptible line 15I birds compared to resistant line $\mathrm{N}$ birds (Fig. 6b) suggesting that IBV infection might cause more disorder of eggshell formation in this line [49]. The production of poor quality eggs by IBV infected birds may, in part be a reflection of the expression of these kinds of gene networks compared to that seen in resistant birds.

\section{Confirmation of differential gene expression by quantitative real-time PCR}

Twenty-one genes were selected for qRT-PCR validation (Table 3). These genes were chosen based on their involvement in the host response and whether they were differentially expressed between the susceptible and resistant lines (either inherently or during the course of infection). Of the 21 genes tested, 19 showed comparable

Table 4 Potential candidate genes for involvement in resistance to IBV

\begin{tabular}{|c|c|c|c|c|}
\hline Gene & Description & Fold change & GenBank & Ensembl \\
\hline$\overline{M X 1}$ & Interferon-induced GTP-binding protein Mx. & $4-24^{a}$ & NM_204609 & ENSGALG00000016142 \\
\hline C1S & Complement component 1, s subcomponent & $2^{a}$ & NM_001030777 & ENSGALG00000014603 \\
\hline IRF7 & Interferon regulatory factor 7 (IRF-7). & $6^{a}$ & NM_205372 & ENSGALG00000014297 \\
\hline TLR3 & Toll-like receptor 3 & $4^{a}$ & NM_001011691 & ENSGALG00000013468 \\
\hline C1R & Complement component $1, \mathrm{r}$ subcomponent & $2^{\mathrm{a}}$ & XM_416518 & ENSGALG00000014659 \\
\hline CCLi7 & Chemokine ah221 & $8^{\mathrm{a}}$ & AY037860 & ENSGALG00000002343 \\
\hline ISG12-2 & Interferon stimulated gene $12-2$ & $16-18^{\mathrm{a}}$ & NM_001001296 & ENSGALG00000013575 \\
\hline IFITM3 & Interferon induced transmembrane protein 3 & $4-5^{a}$ & KC876032 & ENSGALG00000004243 \\
\hline CD38 & ADP-ribosyl cyclase 1 & $4^{\mathrm{b}}$ & XM_420774 & ENSGALG00000014508 \\
\hline CD4 & CD4 protein & $4^{\mathrm{b}}$ & NM_204649 & ENSGALG00000014477 \\
\hline FKBP5 & FK-506 binding protein 51 & $2^{\mathrm{b}}$ & NM_001005431 & ENSGALG00000000947 \\
\hline STAT3 & Signal transducer and activator of transcription 3 (acute-phase response factor) & $3^{\mathrm{b}}$ & NM_001030931 & ENSGALG00000003267 \\
\hline OASL & 2'-5'-oligoadenylate synthetase-like & $4^{c}$ & NM_205041 & ENSGALG00000013723 \\
\hline DDT & D-dopachrome tautomerase & $62-72^{c}$ & NM_001030667 & ENSGALG00000006350 \\
\hline SRI & Sorcin & $65-89^{c}$ & NM_001080865 & ENSGALG00000008985 \\
\hline BLB1 & MHC Class II beta 1 and 2 domains & $10-22^{c}$ & NM_001044694 & ENSGALG00000000141 \\
\hline IFNAR2 & Interferon alpha/beta receptor 2 & $2-3^{c}$ & NM_204858 & ENSGALG00000015938 \\
\hline TPD52L1 & Tumor protein D53 homolog & $12-14^{c}$ & NM_204215 & ENSGALG00000014843 \\
\hline BCL2L1 & BCL2-like - apoptosis regulator & $1.7^{\mathrm{c}}$ & NM_001025304 & ENSGALG00000006211 \\
\hline FAIM2 & Fas apoptotic inhibitory molecule 2 & $1.7^{c}$ & XM_004950568 & ENSGALG00000027555 \\
\hline CIAPIN1 & Cytokine Induced Apoptosis Inhibitor 11 & $1.7^{\mathrm{c}}$ & NM_001005834 & ENSGALG00000005706 \\
\hline $\mathrm{HSCB}$ & Co-chaperone protein $\mathrm{HscB}$, mitochondrial precursor ( $\mathrm{Hsc} 20)$. & $8-10^{c}$ & XR_026662 & ENSGALG00000005706 \\
\hline BF1 & MHC class I antigen B-F minor heavy chain & $13-27^{c}$ & NM_001097530 & ENSGALG00000000178 \\
\hline BF2 & Major class I glycoprotein precursor & $7-8^{c}$ & NM_001031338 & ENSGALG00000000178 \\
\hline SUCLG2 & succinate-CoA ligase, GDP-forming, beta subunit & $16-32^{c}$ & NM_001006141 & ENSGALG00000007652 \\
\hline
\end{tabular}

aupregulated in response to infection in the susceptible line

${ }^{b}$ Higher expression in response to infection in the resistant than in the susceptible line

Inherently higher expression in the resistant line 
differential expression to that determined by the arrays. However, the results for IFNAR2 and IGFBP5 were not confirmed (Additional file 4: Figure S1).

\section{Potential candidate genes for IBV resistance}

Besides knowing that the MHC B locus has a bearing on disease resistance, the lack of any genetic information or identified QTL meant that we had to rely upon the gene expression differences we saw between susceptible and resistant lines to give us clues as to genes potentially involved in resistance to IBV infection. Identifying genes which were expressed at different levels in the two lines of birds highlighted B-locus genes (BLB1, $B F 1, B F 2, B-G)$ as well as bringing to our attention various other non-MHC genes which, due to their known biology, could be candidates for being involved in resistance to IBV infection (Table 4).

MX1, C1S, IRF7, TLR3, C1R, CCLi7, ISG12-2 and IFITM3 are all strongly induced during the host response to IBV infection. They are all innate immune genes which could potentially have a role in determining susceptibility to the virus. MX1 and IFITM3 are already established as anti-viral molecules [50-52]. CD38, CD4, FKBP5 and STAT3 all show a higher level of expression during the host response in the resistant birds compared to that of the susceptible birds, indicating their involvement in the host defence mechanism. CD38 and CD4, with their role as receptors on immune cells, as described above, are obvious candidates, along with FKBP5 as an immune-regulator. STAT3 is activated by various cytokines and growth factors and functions in cellular processes such as cell growth and apoptosis.

Even before infection, many genes are seen to be highly differentially expressed between lines $15 \mathrm{I}$ and N. OASL is an interferon-induced molecule known to have anti-viral activity against certain viruses such as hepatitis $C$ virus. DDT is highly homologous to the macrophage migration inhibition factor, MIF. We have also shown it to be highly differentially expressed in other chicken lines, which are susceptible or resistant to Marek's Disease virus [53]. IFNAR2 is an obvious candidate prediction, as the interferon response is central to the host's defence against IBV infection. TPD52L1, BCL2L1, FAIM2 and CIAPIN1 are all known to be involved in regulation of apoptosis, a process seen to be important during IBV infection. HSCB, SRI, and SUCLG2, although not having an obvious potential biological role in disease resistance, are highly differentially expressed between susceptible and resistant lines and should thus be considered as potential candidates.

\section{Conclusions}

Resistance to IBV infection is brought about by the immune response after the virus has entered the host and is not due to prevention of initial viral infection. There is a small initial innate response at $2 \mathrm{dpi}$, with much more gene expression seen at 3 and 4 dpi. Analysis of genes being activated or inhibited upon infection shows that the biological pathways primarily affected during IBV infection include MAPK signalling, those involved in the interferon response and those involving pattern recognition receptors.

Susceptible and resistant lines show a differential host response mostly at $2 \mathrm{dpi}$. There are also genes which are inherently different between the two lines studied, including many genes, which control the apoptotic potential of the host. These differences seen in gene expression levels, allow us to postulate on many candidate genes for disease resistance. Some potential candidates for involvement in disease resistance include genes already known to confer resistance to other viral infections (MHC-B locus genes, $M X 1, O A S L$ and IFITM3), genes involved in apoptotic processes (TPD52L1, BCL2L1, FAIM2 and CIAPIN1) and others which could be potential candidates due to their known biology (e.g. $D D T$ and $C D 4$ ).

\section{Availability of supporting data}

Array data have been submitted to Array Express (http://www.ebi.ac.uk/arrayexpress/) under the accession number E-TABM-1128.

\section{Additional files}

Additional file 1: Table S1. Gene expression seen during the host response to IBV infection in the trachea of susceptible birds. (XLSX $24 \mathrm{~kb}$ )

Additional file 2: Table S2. Gene expression differences found to be inherent between susceptible and resistant lines in the trachea. (XLS 386 kb)

Additional file 3: Table S3. Genes found to be differentially expressed between susceptible and resistant lines in response to IBV infection in the trachea. (XLS $107 \mathrm{~kb}$ )

Additional file 4: Figure S1. qRT-PCR analysis of 21 genes differentially expressed during IBV infection. (A). MX1 (B). C1S (C). IRF7 (D). TLR3 (E). CCLi7 (F). DDT (G). SRI (H). CLU (I). COX11 (J). IFNAR2 (K). TNFAIP1 (L). TP-D53 (M). MAP4K4 (N). MAFK (O). CCL13 (P). HSC20 (Q). SUCLG2 (R). MMD2 (S). CD38 (T). FK506-BP51 (U). IGFBP5. Graphs A-E show expression changes during the host response in the susceptible line. Graphs F-R show inherent differences in gene expression between susceptible and resistant control birds, while graphs $\mathrm{S}-\mathrm{U}$ indicate differential gene expression in susceptible and resistant lines during the host response. (PDF 97 kb)

\section{Abbreviations}

BSA: Bovine serum albumin; $\mathrm{CID}_{50}$ : mean chicken infectious dose; DE: Differentially expressed; FARMS: Factor Analysis for Robust Microarray Summarization; FDR: False discovery rate; GCOS: GeneChip Operating Software; GO: Gene ontology; IB: Infectious bronchitis; IBV: Infectious bronchitis virus; KEGG: Kyoto Encyclopaedia of Genes and Genomes; MHC: Major histocompatibility complex; OD: Optical density; PBS: Phosphate buffered saline; PLIER: Probe Logarithmic Error Intensity Estimate; qRTPCR: Quantitative reverse transcription polymerase chain reaction; QTL: Quantitative trait loci; RIN: RNA integrity number; SARS: Severe acute respiratory syndrome; SILAC: Stable isotope labelling with amino acids in cell culture; TFBS: Transcription factor binding site.

\section{Competing interests}

The authors declare no conflicts of interest and no competing financial interests. 


\section{Authors' contributions}

JS performed the arrays, analysed the results and wrote the manuscript; DC carried out challenge experiments, J-RS prepared RNA, measured viral load and performed qRT-PCR; DB and PK conceived and supervised the project and revised the manuscript. All authors read and approved the final manuscript.

\section{Acknowledgements}

This work was supported by the Biotechnology and Biological Science Research Council (BBSRC), as part of grant numbers BB/D013704/1, BB/ D013704/2 and BB/D010705/1. The authors would like to thank Alison Downing (Edinburgh Genomics, Edinburgh, UK) for excellent technical assistance with the Affymetrix microarray experiments.

\section{Author details}

${ }^{1}$ The Roslin Institute \& R(D)SVS, University of Edinburgh, Easter Bush, Midlothian EH25 9RG, UK. ${ }^{2}$ The Pirbright Institute, Compton Laboratory, Compton, Berkshire RG20 7NN, UK

Received: 1 July 2015 Accepted: 5 October 2015 Published online: 09 October 2015

\section{References}

1. Schalk AF, Hawn MC. An apparently new respiratory disease in baby chicks. J Am Vet Med Assoc. 1931;78:413-22.

2. Beach JR, Schalm OW. Studies of infectious coryza of chickens with special reference to its etiology. Poult Sci. 1936;15:199-206.

3. Beaudette FR, Hudson CB. Cultivation of the virus of infectious bronchitis. J Am Vet Med Assoc. 1937;90:51-8.

4. Cavanagh D. Coronavirus avian infectious bronchitis virus. Vet Res. 2007;38:281-97.

5. Enjuanes L, Sola I, Zúñiga S, Almazán F. Coronavirus Replication and Interaction with Host. In: Mettenleiter TC, Sobrino F, editors. Animal Viruses: Molecular Biology. Norfolk: Caister Academic Press; 2008. p. 149-202.

6. Casais R, Dove B, Cavanagh D, Britton P. Recombinant avian infectious bronchitis virus expressing a heterologous spike gene demonstrates that the spike protein is a determinant of cell tropism. J Virol. 2003;77:9084-9.

7. Asif M, Lowenthal JW, Ford ME, Schat KA, Kimpton WG, Bean AG. Interleukin-6 expression after infectious bronchitis virus infection in chickens. Viral Immunol. 2007;20:479-86.

8. Jackwood MW. Review of infectious bronchitis virus around the world. Avian Dis. 2012;56:634-41.

9. Meulemans G, Van den Berg TP. Nephropathogenic avian infectious bronchitis viruses. World Poult Sci. 1998;54:145-53.

10. Ariaans MP, Matthijs MG, van Haarlem $D$, van de Haar $P$, van Eck JH, Hensen EJ, et al. The role of phagocytic cells in enhanced susceptibility of broilers to colibacillosis after Infectious Bronchitis Virus infection. Vet Immunol Immunopathol. 2008;123:240-50.

11. Matthijs MG, van Eck JH, Landman WJ, Stegeman JA. Ability of Massachusetts-type infectious bronchitis virus to increase colibacillosis susceptibility in commercial broilers: a comparison between vaccine and virulent field virus. Avian Pathol. 2003:32:473-81.

12. Jackwood MW, de Wit S. Infectious Bronchitis. In: Swayne DE, Glisson JR, McDougald LR, Nolan LK, Suarez DL, Nair VL, editors. Diseases of Poultry. 13th ed. UK: Wiley-Blackwell; 2013. p. 139-60.

13. Worthington KJ, Currie RJW, Jones RC. A reverse transcriptase-polymerase chain reaction survey of infectious IBV infection bronchitis virus genotypes in Western Europe from 2002 to 2006. Avian Pathol. 2008;37:247-57.

14. Ignjatovic J, Gould G, Sapats S. Isolation of a variant infectious bronchitis virus in Australia that further illustrates diversity among emerging strains. Arch Virol. 2006;151:1567-85.

15. Mase M, Kawanishi N, Ootani Y, Murayama K, Karino A, Inoue T. A novel genotype of avian infectious bronchitis virus isolated in Japan in 2009. J Vet Med Sci. 2010;72:1265-8.

16. Banat GR, Tkalcic S, Dzielawa JA, Jackwood MW, Saggese MD, Yates $L$, et al. Association of the chicken MHC B haplotypes with resistance to avian coronavirus. Dev Comp Immunol. 2013:39:430-7.

17. Dar A, Munir S, Vishwanathan S, Manuja A, Griebel P, Tikoo S, et al. Transcriptional analysis of avian embryonic tissues following infection with avian infectious bronchitis virus. Virus Res. 2005;110:41-55.
18. Wang X, Rosa AJM, Oliverira HN, Rosa GJM, Guo X, Travnicek M, et al. Transcriptome of Local Innate and Adaptive Immunity during Early Phase of Infectious Bronchitis Viral Infection. Viral Immunol. 2006;19:768-74.

19. Drosten C, Günther S, Preiser W, van der Werf S, Brodt HR, Becker S, et al. Identification of a novel coronavirus in patients with severe acute respiratory syndrome. N Engl J Med. 2003:348:1967-76.

20. Ksiazek TG, Erdman D, Goldsmith CS, Zaki SR, Peret T, Emery S, et al. A novel coronavirus associated with severe acute respiratory syndrome. N Engl J Med. 2003;348:1953-66.

21. Cook J, Otsuki K, Huggins M, Bumstead N. Investigations into resistance of chicken lines to infection with infectious bronchitis virus. Adv Exp Med Biol. 1990;276:491-6.

22. Stone HA. Use of highly inbred chickens in research. USDA Agricultural Research Service Technical Bulletin. 1975, No.1514, Washington, DC.

23. Cole RK. Studies on genetic resistance to Marek's disease. Avian Dis. 1968;12:9-28.

24. Affymetrix. Guide to Probe Logarithmic Intensity Error (PLIER) Estimation. Santa Clara: Affymetrix I; 2005

25. Talloen W, Clevert DA, Hochreiter S, Amaratunga D, Bijnens L, Kass S, et al. I/NI-calls for the exclusion of non-informative genes: a highly effective filtering tool for microarray data. Bioinformatics. 2007;23:2897-902.

26. R Development Core Team. R: A language and environment for statistical computing. Vienna: R Foundation for Statistical Computing; 2007. http://www.R-project.org. ISBN 3-900051-07-0.

27. Khatri P, Sellamuthu S, Malhotra P, Amin K, Done A, Draghici S. Recent additions and improvements to the Onto-Tools. Nucleic Acids Res. 2005;33(Web Server issue):W762-5.

28. Pathway Express. [http://vortex.cs.wayne.edu/projects.htm]

29. Kanehisa M, Goto S. KEGG: kyoto encyclopedia of genes and genomes. Nucleic Acids Res. 2000;28:27-30.

30. Ingenuity Pathway Analysis. [Ingenuity ${ }^{\oplus}$ Systems. www.ingenuity.com]

31. Expander. [http://acgt.cs.tau.ac.il/expander/expander.html]

32. Eldaghayes I, Rothwell L, Williams A, Withers D, Balu S, Davison F, et al. Infectious bursal disease virus: strains that differ in virulence differentially modulate the innate immune response to infection in the chicken bursa. Viral Immunol. 2006;19:83-91.

33. Poh TY, Pease J, Young JR, Bumstead N, Kaiser P. Re-evaluation of chicken CXCR1 determines the true gene structure: CXCLi1 (K60) and CXCLi2 (CAF) interleukin-8) are ligands for this receptor. J Biol Chem. 2008;283:16408-15.

34. Rothwell L, Hu T, Wu Z, Kaiser P. Chicken interleukin-21 is costimulatory for T cells and blocks maturation of dendritic cells. Dev Comp Immunol. 2012;36:475-82.

35. Guo X, Rosa AJM, Chen D-G, Wang X. Molecular mechanisms of primary and secondary mucosal immunity using avian infectious bronchitis virus as a model system. Vet Immunol Immunopathol. 2008;121:332-43.

36. Draghici S, Khatri P, Tarca AL, Amin K, Done A, Voichita C, et al. A systems biology approach for pathway level analysis. Genome Res. 2007;17:1537-45.

37. Kameka AM, Haddadi S, Kim DS, Cork SC, Abdul-Careem MF. Induction of innate immune response following infectious bronchitis corona virus infection in the respiratory tract of chickens. Virology. 2014:450-451:114-21.

38. Yang N, Ma P, Lang J, Zhang Y, Deng J, JU X, et al. Phosphatidylinositol 4Kinase III $\mathrm{B}$ Is Required for Severe Acute Respiratory Syndrome Coronavirus Spike-mediated Cell Entry. J Biol Chem. 2012;287:8457-67.

39. Favoreel HW, Van de Walle GR, Nauwynck HJ, Pensaert MB. Virus complement evasion strategies. J Gen Virol. 2003;84:1-15.

40. Ariaans MP, van de Haar PM, Hensen EJ, Vervelde L. Infectious Bronchitis Virus induces acute interferon-gamma production through polyclonal stimulation of chicken leukocytes. Virology. 2009;385:68-73.

41. Vervelde L, Matthijs MG, van Haarlem DA, de Wit JJ, Jansen CA. Rapid NKcell activation in chicken after infection with infectious bronchitis virus M41. Vet Immunol Immunopathol. 2013:151:337-41.

42. Kint J, Fernandez-Gutierrez M, Maier HJ, Britton P, Langereis MA, Koumans J, et al. Activation of the chicken type I IFN response by infectious bronchitis coronavirus. J Virol. 2015;89:1156-67.

43. Sharan R, Maron-Katz A, Shamir R. CLICK and EXPANDER: a system for clustering and visualizing gene expression data. Bioinformatics. 2003;19:1787-99.

44. Emmott $E_{1}$ Munday $D$, Bickerton $E$, Britton $P$, Rodgers MA, Whitehouse $A$, et al. The cellular interactome of the coronavirus infectious bronchitis virus nucleocapsid protein and functional implications for virus biology. J Virol. 2013;87:9486-500

45. Crinion RA. Egg quality and production following infectious bronchitis virus exposure at one day old. Poult Sci. 1972;51:582-5. 
46. Brionne A, Nys Y, Hennequet-Antier C, Gautron J. Hen uterine gene expression profiling during eggshell formation reveals putative proteins involved in the supply of minerals or in the shell mineralization process. BMC Genomics. 2014;15:220.

47. Cong F, Liu X, Han Z, Shao Y, Kong X, Liu S. Transcriptome analysis of chicken kidney tissues following coronavirus avian infectious bronchitis virus infection. BMC Genomics. 2013;14:743.

48. Liu C, Xu HY, Liu DX. Induction of caspase-dependent apoptosis in cultured cells by the avian coronavirus infectious bronchitis virus. J Virol. 2001;75:6402-9.

49. Nii T, Isobe N, Yoshimura Y. Effects of avian infectious bronchitis virus antigen on eggshell formation and immunoreaction in hen oviduct. Theriogenology. 2014;81:1129-38.

50. Goodbourn S, Didcock L, Randall RE. Interferons: cell signalling, immune modulation, antiviral response and virus countermeasures. J Gen Virol. 2000;81:2341-64.

51. Haller O, Frese M, Kochs G. Mx proteins: Mediators of innate resistance to RNA viruses. Rev Sci Technol. 1998;17:220-30.

52. Perreira JM, Chin CR, Feeley EM, Brass AL. IFITMs restrict the replication of multiple pathogenic viruses. J Mol Biol. 2013;425:4937-55.

53. Smith J, Sadeyen JR, Paton IR, Hocking PM, Salmon N, Fife M, et al. Systems analysis of immune responses in Marek's disease virus-infected chickens identifies a gene involved in susceptibility and highlights a possible novel pathogenicity mechanism. J Virol. 2011;85:11146-58.

\section{Submit your next manuscript to BioMed Central and take full advantage of:}

- Convenient online submission

- Thorough peer review

- No space constraints or color figure charges

- Immediate publication on acceptance

- Inclusion in PubMed, CAS, Scopus and Google Scholar

- Research which is freely available for redistribution 